\title{
A New Method for Constructing Factorisable Representations for Current Groups and Current Algebras
}

\author{
K. R. Parthasarathy and K. Schmidt \\ Mathematics Institute, University of Warwick, Coventry, Warwickshire CV4 7AL, England
}

\begin{abstract}
Let $C_{e}^{\infty}\left(R^{n}, G\right)$ denote the group of infinitely differentiable maps from $n$-dimensional Euclidean space into a simply connected and connected Lie group, which have compact support. This paper introduces a class of factorisable unitary representations of $C_{e}^{\infty}\left(R^{n}, G\right)$ with the property that the unitary operator $U_{f}$ corresponding to a function $f$ in $C_{e}^{\infty}\left(R^{n}, G\right)$ depends not only on $f$, but also on the derivatives of $f$ up to a certain order. In particular these representations can not be extended to the group of all continuous functions from $R^{n}$ to $G$ with compact support.
\end{abstract}

\section{$\S 1$. Introduction}

Let $G$ be a simply connected and connected Lie group and let $\mathscr{G}$ be its Lie algebra. Let exp: $\mathscr{G} \rightarrow G$ denote the exponential map. We denote by $C_{e}^{\infty}(R, G)$ the class of all $C^{\infty}$ maps from $R$ into $G$ with compact support. A map $\varphi: R \rightarrow G$ is said to have compact support if takes the value $e$, i.e., the identity element of $G$ outside a compact set. Let $C_{0}^{\infty}(R, \mathscr{G})$ denote the class of all infinitely differentiable maps from $R$ into the vector space $\mathscr{G}$ with compact support. For any $f \in C_{0}^{\infty}(R, \mathscr{G})$, we define $\operatorname{Exp} f \in C_{e}^{\infty}(R, G)$ by writing $(\operatorname{Exp} f)(x)=\exp f(x)$, for all $x \in R$. $C_{e}^{\infty}(R, G)$ is a group (under pointwise multiplication) and $C_{0}^{\infty}(R, \mathscr{G})$ is a Lie algebra (under pointwise addition, scalar multiplication and Lie brackets). These may respectively be called as current group and current algebra over $R$. We give $C_{0}^{\infty}(R, \mathscr{G})$ the usual Schwarz topology. A homomorphism $\varphi \rightarrow U_{\varphi}$ of the group $C_{e}^{\infty}(R, G)$ into the group of unitary operators on a Hilbert space $H$ is said to be a unitary representation or simply a representation if $U_{\operatorname{Exp} f_{n}}$ converges weakly to $U_{\operatorname{Exp} f}$ whenever $f_{n} \rightarrow f$ as $n \rightarrow \infty$ in the topology of $C_{0}^{\infty}(R, \mathscr{G})$.

For any compact set $K \subset R$, let $C(K, G) \subset C_{0}^{\infty}(R, G)$ be the subgroup of all those maps with support contained in $K$. If $K_{1}, K_{2}$ are two disjoint compact subsets of $R, C\left(K_{1} \cup K_{2}, G\right)$ can be identified in a natural manner with the cartesian product $C\left(K_{1}, G\right) \times C\left(K_{2}, G\right)$. Indeed, for any $\varphi \in C\left(K_{1} \cup K_{2}, G\right)$, define

$$
\begin{aligned}
\varphi_{i}(x) & =\varphi(x) \quad \text { if } \quad x \in K_{i} \\
& =e \quad \text { if } \quad x \notin K_{i}, \quad i=1,2 .
\end{aligned}
$$


Then $\varphi=\varphi_{1} \varphi_{2}$. The map $\varphi \rightarrow\left(\varphi_{1}, \varphi_{2}\right)$ gives the required identification. For any representation $U$ of $C_{e}^{\infty}(R, G)$, we define the representation $U^{K}$ of the subgroup $C(K, G)$ by

$$
U_{\varphi}^{K}=U_{\varphi}, \varphi \in C(K, G) .
$$

We say that a representation $U$ of $C_{e}^{\infty}(R, G)$ is factorisable if, for any two disjoint compact sets $K_{1}, K_{2} \subset R$, the representation $U^{K_{1} \cup K_{2}}$ is unitarily equivalent to the tensor product $U^{K_{1}} \otimes U^{K_{2}}$. This unitary equivalence will of course depend on $K_{1}$ and $K_{2}$. Examples of such factorisable representations based on the unitary representations of $G$ and their first cohomologies were first constructed by Streater [6] and Araki [1]. Further development of these ideas may be found in the works of Parthasarathy and Schmidt [4, 3], Vershik, Gelfand and Graev [7], and Guichardet [2]. However, most of these examples have the degenerate property that they factorise completely. These representations extend to borel maps from $R$ into $G$ and the factorisability property extends to pairs of disjoint borel sets. This is mainly because the representations constructed in these papers do not involve the derivatives of smooth maps in a certain sense. One may compare this with the following situation in the classical theory of distributions. To evaluate the Dirac $\delta$ at a testing function $\varphi$ one need not know the derivations of $\varphi$. However to evaluate the distributions $\delta^{\prime}, \delta^{\prime \prime}, \ldots$ one requires a knowledge of $\varphi^{\prime}, \varphi^{\prime \prime}, \ldots$. The main aim of this paper is to construct factorisable representations $U$ which for their evaluation at $\operatorname{Exp} f, f \in C_{0}^{\infty}(R, \mathscr{G})$ requires a knowledge of $f, f^{\prime}, f^{\prime \prime}, \ldots$ A beginning in this direction was already made by Schmidt [5] in the case when $G$ is the Heisenberg group, whose representations lead to canonical commutation relations.

\section{§ 2. The Leibnitz Extension of a Lie Algebra}

In order to outline the method of constructing factorisable representations we need to construct an extension of the Lie algebra $\mathscr{G}$. To this end consider the space $\mathscr{G}_{n}$ which is the $n+1$-fold Cartesian product of $\mathscr{G}$. Any element $\boldsymbol{X}$ of $\mathscr{G}_{n}$ can be written as

$$
\boldsymbol{X}=\left(X_{0}, X_{1}, \ldots, X_{n}\right), X_{i} \in \mathscr{G} \text { for each } i \text {. }
$$

Between two elements $\boldsymbol{X}$ and $\boldsymbol{X}^{\prime}$ in $\mathscr{G}_{n}$ define the bracket operation by

$$
\left[X, X^{\prime}\right]=X^{\prime \prime},
$$

where

$$
\begin{aligned}
& X_{0}^{\prime \prime}=\left[X_{0}, X_{0}^{\prime}\right], \\
& X_{j}^{\prime \prime}=\sum_{r=0}^{j}\left(\begin{array}{l}
j \\
r
\end{array}\right)\left[X_{r}, X_{j-r}^{\prime}\right] .
\end{aligned}
$$

An easy computation shows that for $\boldsymbol{X}, \boldsymbol{Y}, \boldsymbol{Z} \in \mathscr{G}_{n}$,

$$
[[X, Y] Z]=T
$$


where

$$
T_{r}=\sum_{k_{1}+k_{2}+k_{3}=r}\left(r ! / k_{1} ! k_{2} ! k_{3} !\right)\left[\left[X_{k_{1}}, Y_{k_{2}}\right], Z_{k_{3}}\right] .
$$

This shows that

$$
[[\boldsymbol{X}, \boldsymbol{Y}], \boldsymbol{Z}]+[[\boldsymbol{Y}, \boldsymbol{Z}], \boldsymbol{X}]+[[\boldsymbol{Z}, \boldsymbol{X}], \boldsymbol{Y}]=0 .
$$

In other words $\mathscr{G}_{n}$ becomes a Lie algebra. We shall call $\mathscr{G}_{n}$ the $n^{\text {th }}$ Leibnitz extension of the Lie algebra $\mathscr{G}$. The mapping $X \rightarrow(X, 0,0, \ldots, 0)$ is an isomorphism of $\mathscr{G}$ into $\mathscr{G}_{n}$. All elements of the form $\left(0, X_{1}, X_{2}, \ldots, X_{n}\right), X_{1} \in \mathscr{G}, i=1,2 \ldots n$ constitute a nilpotent Lie subalgebra $h^{(n)}$ of $\mathscr{G}_{n}$. Further

$$
\begin{aligned}
& {\left[(X, 0,0, \ldots, 0),\left(0, X_{1}, X_{2}, \ldots, X_{n}\right)\right]} \\
& =\left(0,\left[X, X_{1}\right],\left[X, X_{2}\right], \ldots,\left[X, X_{n}\right]\right) .
\end{aligned}
$$

Thus $\mathscr{G}$ acts as a Lie algebra of derivations of the nilpotent Lie algebra $h^{(n)}$. In other words $\mathscr{G}_{n}$ is a semi-direct sum of $\mathscr{G}$ and $h^{(n)}$.

Remark 2.1. Since any Lie algebra $\mathscr{G}$ can be represented as a Lie algebra of matrices, we shall assume that $\mathscr{G}$ is a Lie algebra of real matrices in all our computations hereafter. Let the order of the matrices in $\mathscr{G}$ be $k \times k$.

Lemma 2.2. The map

$$
A:\left(0, X_{1}, X_{2}, \ldots, X_{n}\right) \rightarrow A\left(X_{1}, X_{2}, \ldots, X_{n}\right), X_{i} \in \mathscr{G}, i=1,2 \ldots n
$$

where

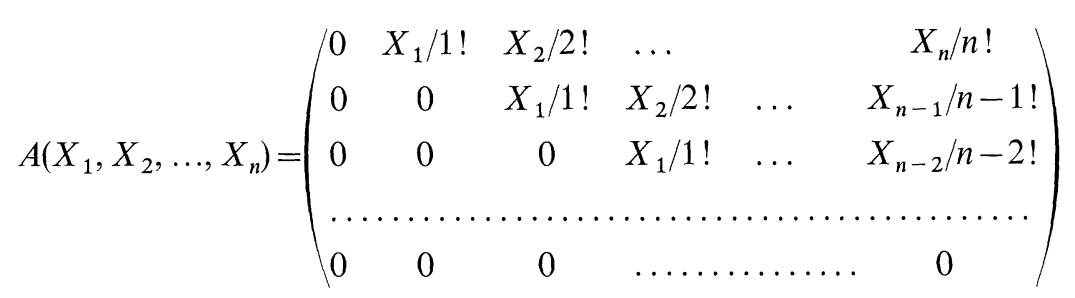

is an isomorphism of the Lie algebra $h^{(n)}$ into the Lie algebra of all matrices of order $k(n+1) \times k(n+1)$.

Proof. This follows from a routine computation and is left to the reader.

Lemma 2.3. Let $A$ be the map defined in the preceding lemma. Then the matrix $\exp A\left(X_{1}, X_{2}, \ldots, X_{n}\right)$ is of the form

$$
\left(\begin{array}{cccccc}
I & A_{1} & A_{2} & \ldots & \ldots & A_{n} \\
0 & I & A_{1} & A_{2} & \ldots & A_{n-1} \\
0 & 0 & I & A_{1} & \ldots & A_{n-2} \\
\ldots \ldots \ldots \ldots \ldots \ldots & \ldots \ldots \ldots \\
0 & 0 & 0 & 0 & \ldots & I
\end{array}\right)
$$


where

$$
A_{j}=\sum_{p=1}^{j} 1 / p ! \sum_{\substack{m_{1}+\ldots+m_{p}=j \\ 1 \leqq m_{i} \leqq j}} m_{1} !^{-1} X_{m_{1}} m_{2} !^{-1} X_{m_{2}} \ldots m_{p} !^{-1} X_{m_{p}} .
$$

Proof. It is left to the reader.

Remark 2.4. Let $H$ be the group generated (algebraically) by all matrices of the form $\exp A\left(X_{1}, X_{2}, \ldots, X_{n}\right), X_{i} \in \mathscr{G}, i=1,2 \ldots n$. Its Lie algebra is isomorphic with $h^{(n)}$. Let $G$ be the simply connected group for which the Lie algebra is $\mathscr{G}$. Then for any $X_{0} \in \mathscr{G}$, the element $\exp X_{0}$ of $G$ acts as an automorphism of $H$ as follows:

$$
\begin{aligned}
& \exp X_{0}: \exp A\left(X_{1}, X_{2}, \ldots, X_{n}\right) \\
& \rightarrow \exp A\left(e^{\operatorname{ad} X_{0}}\left(X_{1}\right), e^{\operatorname{ad} X_{0}}\left(X_{2}\right), \ldots, e^{\operatorname{ad} X_{0}}\left(X_{n}\right)\right) .
\end{aligned}
$$

Hence we can form the semi-direct product $G \odot H$ of the two groups $G$ and $H$. $G \odot H$ consists of all pairs $(g, h), g \in G, h \in H$. The multiplication operation is defined by

$$
(g, h) \cdot\left(g^{\prime}, h^{\prime}\right)=\left(g g^{\prime}, h \cdot g\left(h^{\prime}\right)\right),
$$

where $h^{\prime} \rightarrow g\left(h^{\prime}\right)$ is the automorphism of $H$ induced by $g$. The Lie algebra of the group $G \odot H$ is then isomorphic to the Lie algebra $\mathscr{G}_{n}$. In particular $\mathscr{G}_{1}$ is the Lie algebra of the semidirect product of $G$ and the additive group $\mathscr{G}$, where $G$ acts as the adjoint representation in $\mathscr{G}$.

Lemma 2.4. For any $\boldsymbol{X}=\left(X_{0}, X_{1}, \ldots, X_{n}\right) \in \mathscr{G}_{n}$, the exponential map from $\mathscr{G}_{n}$ into $G \odot H$ is defined as follows: let

$$
A_{j}(t)=\sum_{p=1}^{j} \sum_{\substack{m_{1}+\ldots+m p=j \\ 1 \leqq m_{l} \leqq j}} \int_{0<t_{1}<t_{2}<\ldots<t_{p}<t}\left(\prod_{k=1}^{p} e^{t_{k} \text { ad } X_{0}}\left(m_{k} !^{-1} X_{m_{k}}\right)\right) d t_{1} \ldots d t_{p}
$$

for $j=1,2 \ldots n$. Let

$$
A(t)=\left(\begin{array}{ccccc}
I & A_{1}(t) & A_{2}(t) & \ldots A_{n}(t) \\
0 & I & A_{1}(t) & \ldots & A_{n-1}(t) \\
0 & 0 & I & \ldots & A_{n-2}(t) \\
\ldots \ldots \ldots \ldots \ldots \ldots \ldots \ldots & \ldots \ldots & \ldots & \ldots
\end{array}\right)
$$

Then

$$
\exp t \boldsymbol{X}=\left(\exp t X_{0}, A(t)\right) \quad \text { for all } \quad t \in R .
$$

Proof. Indeed, differentiating (2.2) at $t=0$, we get

$$
d A_{j} /\left.d t\right|_{t=0}=j !^{-1} X_{j} .
$$


Thus

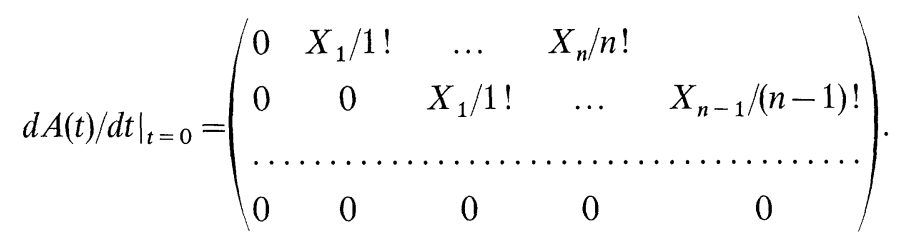

Further

$$
\begin{aligned}
& \left(\exp t X_{0}, A(t)\right) \cdot\left(\exp s X_{0}, A(s)\right) \\
& =\left(\exp (t+s) X_{0}, A(t) \cdot \exp t X_{0}(A(s)),\right.
\end{aligned}
$$

where

$$
\exp t X_{0}(A(s))=\left(\begin{array}{ccccc}
I & B_{1} & B_{2} & \ldots & B_{n} \\
0 & I & B_{1} & \ldots & B_{n-1} \\
\ldots & \ldots & \ldots & \ldots & \ldots \\
0 & 0 & 0 & \ldots & I
\end{array}\right)
$$

and

$$
\begin{aligned}
B_{j} & =B_{j}(t, s)=e^{t X_{0}} A_{j}(s) e^{-t X_{0}} \\
& =\sum_{p=1}^{j} \sum_{\substack{m_{1}+\ldots+m_{p}=j \\
m_{2} \geqq 1 \text { for alli }}} \int_{0<t_{1}<t_{2}<\ldots<t_{p}<s} \prod_{k=1}^{p} e^{\left(t_{k}+t\right) \operatorname{ad} X_{0}}\left(k !^{-1} X_{m_{k}}\right) d t_{1} \ldots d t_{p} \\
& =\sum_{p=1}^{j} \sum_{\substack{m_{1}+\ldots+m_{p}=j \\
m_{2} \geqq 1 \text { for all } i}} \int_{0<t_{1}<t_{2}<\ldots<t_{p}<t+s} \prod_{k=1}^{p} e^{t_{k} \text { ad } X_{0}}\left(k !^{-1} X_{m_{k}}\right) d t_{1} \ldots d t_{p} .
\end{aligned}
$$

A straightforward matrix multiplication shows that

$$
A(t) \cdot \exp t X_{0}(A(s))=\left(\begin{array}{ccccc}
I & C_{1} & C_{2} & \ldots & C_{n} \\
0 & I & C_{1} & \ldots & C_{n-1} \\
\ldots \ldots & \ldots \ldots & \ldots & \ldots \\
0 & 0 & 0 & \ldots & I
\end{array}\right)
$$

where

$$
\begin{aligned}
C_{j} & =\sum_{r=0}^{j} A_{r}(t) B_{j-r}(t, s), \\
A_{0}(t) & =B_{0}(t, s)=I,
\end{aligned}
$$

and where $A_{r}$ and $B_{r}$ is defined by (2.2) and (2.3) respectively. Now an easy computation gives $C_{j}=A_{j}(t+s)$. This shows that $\left(\exp t X_{0}, A(t)\right)$ is a one parameter group with the generator $\left(X_{0}, X_{1}, X_{2}, \ldots, X_{n}\right)$. The proof is complete. 
Corollary 2.5. When $n=1$ and $G \odot H$ is identified with the semidirect product of $G$ and the additive group $\mathscr{G}$, where $G$ acts as adjoint representation in $\mathscr{G}$, we have

$$
\operatorname{expt}\left(X_{0}, X_{1}\right)=\left(\exp t X_{0}, \frac{e^{t \operatorname{ad} X_{0}-1}}{t \operatorname{ad} X_{0}}\left(X_{1}\right)\right)
$$

for all $t \in R$.

Proof. This follows from the preceding lemma by noting that

$$
\int_{0}^{t} e^{t_{1} \operatorname{ad} X_{0}}\left(X_{1}\right) d t_{1}=\frac{e^{t \operatorname{ad} X_{0}}-1}{t \operatorname{ad} X_{0}}\left(X_{1}\right) .
$$

\section{$\S 3$. Representation of Current Algebras and Current Groups}

In the preceding section we gave a complete description of the group associated with the $n$-th Leibnitz extension $\mathscr{G}_{n}$ of a Lie algebra $\mathscr{G}$. The following lemma yields the required embedding of $C_{0}^{\infty}(R, \mathscr{G})$ into $C_{0}^{\infty}\left(R, \mathscr{G}_{n}\right)$ for writing down our representations.

Lemma 3.1. Let $\Pi_{n}$ be the map from $C_{0}^{\infty}(R, \mathscr{G})$ into $C_{0}^{\infty}\left(R, \mathscr{G}_{n}\right)$ defined by

$$
\Pi_{n}(f)(x)=\left(f(x), f^{\prime}(x), f^{\prime \prime}(x), \ldots, f^{(n)}(x)\right)
$$

for all $x \in R, f \in C_{0}^{\infty}(R, \mathscr{G})$.

Then $\Pi_{n}$ is a Lie algebra isomorphism of $C_{0}^{\infty}(R, \mathscr{G})$ into $C_{0}^{\infty}\left(R, \mathscr{G}_{n}\right)$.

Proof. This follows immediately from the fact that

$$
d^{j}[f, g] / d x^{j}=\sum_{r=0}^{j}\left(\begin{array}{l}
j \\
r
\end{array}\right)\left[f^{(r)}(x), g^{(j-r)}(x)\right]
$$

and the commutation rules in $\mathscr{G}_{n}$ are defined by (2.1).

As mentioned in $\S 1$, we define for any $f \in C_{0}^{\infty}(R, \mathscr{G}), \operatorname{Exp} f$ as the element in $C_{e}^{\infty}(R, G)$ with the property

$$
(\operatorname{Exp} f)(x)=\exp f(x), x \in R \text {. }
$$

Consider the group $G \odot H$ described in Remark 2.4. We shall call it the $n$-th Leibnitz extension of the group $G$. For any $f \in C_{0}^{\infty}(R, \mathscr{G})$, we define $\operatorname{Exp}_{n} f$ as the element in $C_{e}^{\infty}(R, G \odot H)$ with the property

$$
\left(\operatorname{Exp}_{n} f\right)(x)=\left(\exp f(x), A^{f}(x)\right)
$$

where

$$
A^{f}(x)=\left(\begin{array}{ccccc}
I & A_{1}^{f}(x) & A_{2}^{f}(x) & \ldots & A_{n}^{f}(x) \\
0 & I & A_{1}^{f}(x) & \ldots & A_{n-1}^{f}(x) \\
\ldots \ldots \ldots \ldots \ldots \ldots \ldots \ldots \ldots \ldots \\
0 & 0 & 0 & \ldots & I
\end{array}\right)
$$




$$
\begin{aligned}
A_{j}^{f}(x)= & \sum_{p=1}^{j} \sum_{\substack{m_{1}+\ldots+m_{p}=j \\
m_{i} \geqq 1 \text { for all } i \\
0<t_{1}<t_{2}<\ldots<t_{p}<1}} \prod_{k=1}^{p} e^{t_{k} \text { ad } f(x)} \\
& \cdot m_{k} !^{-1} f^{\left(m_{k}\right)}(x) d t_{1} d t_{2} \ldots d t_{p},
\end{aligned}
$$

for $j=1,2 \ldots n$. With this notation we have the following corollary to Lemma 3.1.

Theorem 3.2. Let $G$ be a connected and simply connected Lie group whose $n$-th Leibnitz extension is $G_{n}$. Suppose $\varphi \rightarrow U_{\varphi}$ is a factorisable representation of the current group $C_{e}^{\infty}\left(R, G_{n}\right)$. Then the map

$$
U^{(n)}: \operatorname{Exp} f \rightarrow U_{\operatorname{Exp}_{n} f}, f \in C_{0}^{\infty}(R, \mathscr{G})
$$

determines a factorisable representation of the current group $C_{e}^{\infty}(R, G)$. In particular this determines a factorisable representation of the current algebra $C_{0}^{\infty}(R, \mathscr{G})$.

Remark 3.3. To construct a factorisable representation $U$ of the current group $C_{e}^{\infty}\left(R, G_{n}\right)$ one may start with a projection valued measure on the Borel subsets of $R$, a unitary represention $V$ of the group $G_{n}$ commuting with the projection valued measure and a first order cocycle for the representation $V$, and adopt the procedure outlined in [4]. Since $G$ is a subgroup of $G_{n}$ it follows that $C_{e}^{\infty}(R, G)$ is a subgroup of $C_{e}^{\infty}\left(R, G_{n}\right)$. Hence the restriction of $U$ to $C_{e}^{\infty}(R, G)$ yields a representation $U^{(0)}$ of $C_{e}^{\infty}(R, G)$. The representation $U^{(n)}$ of Theorem 3.1 obtained from $U$ may be considered as the $n$-th derivative of the representation $U^{(0)}$.

Example 3.4. We shall now illustrate the procedure outlined in the preceding remark in a special case. Let $G$ be a compact, connected, simply connected and semi-simple Lie group with Lie algebra $\mathscr{G}$ and Cartan Killing form $B(X, Y)$, $X, Y \in \mathscr{G}$. Let $g \rightarrow \operatorname{Ad} g$ be the adjoint representation of $G$ acting in $\mathscr{G}$. Let $G_{1}$ denote the first Leibnitz extension of $G$. Then $G_{1}$ is the semi direct product of $G$ and the additive group $\mathscr{G}$ in which $G$ acts as a group of automorphisms through the adjoint representation. Any element of $G_{1}$ can be expressed as a pair $(g, X), g \in G$, $X \in \mathscr{G}$. Then $(g, X) \rightarrow \operatorname{Ad} g$ is an irreducible unitary representation $U$ of $G_{1}$ acting in the vector space $\mathscr{G}$ with the positive definite inner product $-B$. Define the map $\delta: G_{1} \rightarrow \mathscr{G}$ by

$$
\delta(g, X)=X .
$$

Then $\delta$ is a first order cocycle for the representation $U$. Hence the function

$$
\Phi(g, X)=\exp \frac{1}{2} B(X, X)
$$

is an infinitely divisible positive definite function on the group $G_{1}$.

Let now $\varphi: R \rightarrow \mathscr{G}$ be a $C_{0}^{\infty}$ map from $R$ into $\mathscr{G}$. Then the map $t \rightarrow\left(\varphi(t), \varphi^{\prime}(t)\right)$ is a $C_{0}^{\infty}$ map from $R$ into $\mathscr{G}_{1}$ the Lie algebra of $G_{1}$. Let

$$
\psi(t)=\frac{e^{\mathrm{ad} \varphi(t)}-1}{\operatorname{ad} \varphi(t)}\left(\varphi^{\prime}(t)\right),
$$

and let

$$
K(\operatorname{Exp} \varphi)=\exp \frac{1}{2} \int B(\psi(t), \psi(t)) d t .
$$


Then $K$ is an infinitely divisible positive definite functional on $C_{e}^{\infty}(R, G)$ which extends to $C_{e}^{1}(R, G)$, the group of all $C^{1}$ maps from $R$ into $G$ with compact support. This positive definite functional defines a factorisable representation of $C_{e}^{1}(R, G)$ which cannot be extended to all bounded borel maps from $R$ into $G$ with compact support.

Since the factorisable representation corresponding to (3.2) is in a sense a continuous tensor product of copies of the irreducible adjoint representation of $G$ one is tempted to conjecture that (3.2) yields an irreducible factorisable representation of $C_{e}^{1}(R, G)$.

Remark 3.5. The theory outlined above extends in a natural manner when $R$ is replaced by $R^{m}$ and one considers current groups $C_{e}^{\infty}\left(R^{m}, G\right)$. To describe this extension we adopt the following conventions. Let, for any positive integer $N$, $F_{N}$ denote the set of all ordered $m$-tuples $j=\left(j_{1}, j_{2}, \ldots, j_{m}\right)$ of non-negative integers such that $j_{1}+j_{2}+\ldots+j_{m}<N$. For any $j \in F_{N}$, let $j !=j_{1} ! j_{2} ! \ldots j_{m} !$, where $0 !=1$. A general point of $R^{m}$ will be denoted by $x=\left(x_{1}, x_{2}, \ldots, x_{m}\right)$. Let $|j|=j_{1}+j_{2}+\ldots+j_{m}$. For any $C^{\infty}$ map $f$ from $R^{m}$ into the Lie algebra $\mathscr{G}$, let

$$
f^{(\underline{j})}=\partial|\underline{j}| f / \partial x_{1}^{j_{1}} \partial x_{2}^{j_{2}} \ldots \partial x_{m}^{j_{m}} .
$$

We now define the $N$-th Leibnitz extension $\mathscr{G}_{N}$ of $\mathscr{G}$ as the set of all maps $\boldsymbol{X}$ from $F_{N}$ into $\mathscr{G}$ with Lie bracket $[\boldsymbol{X}, \boldsymbol{Y}]$ defined by

$$
[X, Y](j)=\sum(j ! / \underline{r} !(j-\underline{r}) ![X(\underline{r}), Y(j-\underline{r})]
$$

where the summation is over all $\underline{0} \leqq \underline{r} \leqq j$. Here $\underline{r} \leqq j$ means that $r_{i} \leqq j_{i}$ for all $i=$ $1,2, \ldots, m$. Then $\mathscr{G}_{N}$ is a Lie algebra. As before $\mathscr{G}$ may be embedded in $\mathscr{G}_{N}$ by mapping any $X \in \mathscr{G}$ to the element $\boldsymbol{X}$ with $\boldsymbol{X}(\underline{0})=X, X(\underline{i})=0$ for $\underline{i} \neq 0$. Let us say that $\underline{i}<j$ if $i \neq j$ but $i \leqq j$. As before all elements $X$ such that $\boldsymbol{X}(\underline{0})=0$ constitute a nilpotent Lie subalgebra $h^{(N)}$ of $\mathscr{G}_{N} \cdot \mathscr{G}_{N}$ is a semidirect sum of $\bar{G}_{\text {and }} h^{(N)}$. For $\boldsymbol{X} \in h^{(N)}$, we define the matrix $A(X)$ whose $(i, j)^{\text {th }}$ element is $X(i+j)$ if $j>i$ and 0 otherwise. The order of the matrix is $c k \times c k$ where $c$ is the cardinality of $F_{N}$ and $k$ is the order of the matrices which constitute the Lie algebra $\mathscr{G}$. Lemma 2.3 now holds with the convention

$$
A_{\underline{j}}=\sum_{p=1}^{|j|} p !^{-1} \sum_{\underline{m}_{1}+\ldots+\underline{m}_{p}=j} \underline{m}_{1} !^{-1} \boldsymbol{X}\left(\underline{m}_{1}\right) \ldots \underline{m}_{p} !^{-1} \boldsymbol{X}\left(\underline{m}_{p}\right) .
$$

Lemma 2.4 holds with the condition

$$
\begin{aligned}
A_{\underline{j}}(t)= & \sum_{p=1}^{|j|} \sum_{\underline{m}_{1}+\ldots+\underline{m}_{p}=\underline{j}} \underbrace{}_{0<t_{1}<t_{2} \ldots<t_{p}<t} \prod_{i=1}^{p} e^{t_{i} \text { ad } \boldsymbol{X}(0)} \\
& \cdot\left(\underline{m}_{i} !^{-1} \boldsymbol{X}\left(\underline{m}_{i}\right)\right) d t_{1} \ldots d t_{p} .
\end{aligned}
$$


Then Theorem 3.2 holds with the condition that in defining the map $f \rightarrow \operatorname{Exp}_{n} f$ we change (3.1) to

$$
\begin{aligned}
A_{\underline{j}}^{f}= & \sum_{p=1}^{\lfloor\underline{j} \mid} \sum_{\underline{m}_{1}+\ldots+\underline{m}_{p}=\underline{j}} \int_{0<t_{1}<t_{2} \ldots<t_{p}<1} \\
& \prod_{i=1}^{p} e^{t_{i} \text { ad } f(x)}\left(\underline{m}_{i} !^{-1} f^{\left(\underline{m}_{v}\right)}(x)\right) d t_{1} \ldots d t_{p} .
\end{aligned}
$$

Acknowledgement. The first named author wishes to thank the Mathematics Institute, University of Warwick and the Science Research Council (U.K.) for their generous assistance in the preparation of this article.

\section{References}

1. Araki,H.: Factorisable representations of current algebra, Publications of R.I.M.S. Kyoto University, Ser. A, 5 (3), 361-422 (1970)

2. Guichardet, A.: Symmetric Hilbert spaces and related topics. In: Lecture Notes in Mathematics, Vol. 261. Berlin-Heidelberg-New York: Springer 1972

3. Parthasarathy, K.R., Schmidt, K.: Positive definite kernels, continuous tensor products, and central limit theorems of probability theory. In: Lecture Notes in Mathematics, Vol. 272. BerlinHeidelberg-New York: Springer 1972

4. Parthasarathy, K.R., Schmidt, K.: Factorisable representations of current groups and the ArakiWoods imbedding theorem. Acta Math. 128, 53-71 (1972)

5. Schmidt, K.: Algebras with quasilocal structure and factorisable representations, Mathematics of Contemporary Physics (ed. R. F. Streater), pp. 237-251. New York: Academic Press 1972

6. Streater, R.F.: Current commutation relations, continuous tensor products and infinitely divisible group representations. Rend. Sci. Int. Fisica E. Fermi, XI, 247-263 (1969)

7. Vershik, A. M., Gelfand,I.M., Graev, M.I.: Representations of the group $S L(2, R)$ where $R$ is a ring of functions. Russ. Math. Surv. 28, 87-132 (1973)

Communicated by H. Araki

Received July 16, 1975; in revised form March 30, 1976 
